\title{
Analysis of beam wander effects for a horizontal-path propagating Gaussian-beam wave: focused beam case
}

\author{
Jaume Recolons \\ Universitat Politècnica de Catalunya \\ Department of Signal Theory \\ and Communications \\ C/Sor Eulàlia d'Anzizu S/N \\ 08034 Barcelona, Spain
}

\author{
Larry C. Andrews, FELLOW SPIE \\ University of Central Florida \\ Department of Mathematics and Florida Space \\ Institute (FSI) \\ Orlando, Florida 32816
}

Ronald L. Phillips, FELLOW SPIE

University of Central Florida

Department of Electrical and Computer

Engineering and Florida Space Institute (FSI)

Orlando, Florida 32816

\begin{abstract}
Failure of the first-order Rytov approximation to properly predict the scintillation index of a large-aperture focused beam, or an uplink collimated (or focused) beam, has been discussed in several recent publications, which cite beam wander effects as the main reason for this failure. We use computer simulations to examine several aspects of beam wander phenomena on a propagating convergent beam in the weak-fluctuation regime over a horizontal path at high altitude for which the refractive index structure parameter is on the order of $C_{n}^{2}=1.39$ $\times 10^{-16} \mathrm{~m}^{-2 / 3}$. Simulation results are presented at various ranges up to $10 \mathrm{~km}$ for (1) the beam wander centroid displacement, (2) the kurtosis excess of the irradiance profile, (3) the irradiance profile, (4) the meansquare hot spot displacement from the boresight and from the centroid, and (5) the scintillation index at the optical axis of the beam. In addition, simulation results are compared with theoretical models. () 2007 Society of Photo-Optical Instrumentation Engineers. [DOI: 10.1117/1.2772263]
\end{abstract}

Subject terms: atmospheric optics; turbulence; beams.

Paper 060777R received Oct. 5, 2006; revised manuscript received Mar. 1, 2007; accepted for publication Mar. 3, 2007; published online Aug. 24, 2007.

\section{Introduction}

First-order, weak fluctuation Rytov theory predicts that the longitudinal component (or on-axis scintillation index) of a beam focused at a fixed distance along a horizontal path continually decreases as the size of the laser transmitter aperture increases. However, this assertion has never been supported by either experimental data or simulation results. In fact, it has been known for a long time that the observed scintillation index of a large-aperture focused beam can be much greater than that predicted by Rytov theory as a result of beam wander effects. ${ }^{1-4}$

In a recent paper, Baker ${ }^{5}$ discussed the preceding discrepancy of the Rytov method for beam-wave scintillation in terms of an asymptotic region called the $D_{1}$ region (see Sec. 4.2), first identified by Charnotskii. ${ }^{6}$ Baker showed that the strict conditions of weak turbulence scintillation demanded by the Rytov method ${ }^{7}$ exclude the $D_{1}$ region, namely, $\sigma_{R}^{2} \ll 1$ and $\left(2 W_{0} / r_{0}\right)^{5 / 3} \ll 1$. Here, $\sigma_{R}^{2}$ is the Rytov variance, $W_{0}$ is the beam radius at the transmitter, and $r_{0}$ is Fried's parameter. Based on this observation, Baker was able to define the borders of the $D_{1}$ region by two dimensionless Fresnel parameters, rather than the three parameters used by Charnotskii.

The current paper is a further analysis of the weak turbulence scintillation associated with beam wander effects of a convergent beam in the vicinity of its geometric focus, which corresponds to the $D_{1}$ region. Special consideration is given to those beams focused at the end of the

0091-3286/2007/\$25.00 @ 2007 SPIE propagation path and those focused at some intermediate point along the path. We report on simulation results involving beam wander-induced scintillation along a $10-\mathrm{km}$ path at high altitude, similar to the propagation path for a free-space optical (FSO) communication link between two aircraft.

In addition to examination of the increase in scintillation caused by beam wander, we also present some simulation results for movement of the beam centroid and beam hot spot (defined later). In that regard, this is a companion paper to our treatment of beam wander phenomena associated with a collimated beam. ${ }^{8}$ Deviation of the irradiance profile from a pure Gaussian shape is described by the coefficient of kurtosis excess. For instance, the kurtosis excess of a Gaussian-shaped irradiance profile is zero, but we find that the kurtosis excess of the short-term and long-term beam is much greater than unity within the $D_{1}$ region, thus providing another characteristic associated with this asymptotic regime. Of special significance is the relation between kurtosis excess and the increase in scintillation in the $D_{1}$ region. Last, the simulation results for scintillation and other beam wander effects are compared with theoretical expressions.

\section{Simulations}

The wave optics code we use is based on the split-step technique, in which the light waves are propagated in the transformed domain and the effect of atmospheric turbulence along the propagation path is simulated by a series of uncorrelated random phase screens. These phase screens are generated under the Markov approximation in the 
spectral domain by generating a set of Gaussian-distributed random numbers with zero mean and variances given by $\left\langle\left|A_{\mu \nu}\right|^{2}\right\rangle=2 \pi k^{2} \Delta z \Phi_{n}\left(\boldsymbol{\kappa}_{\mu \nu}\right)(\Delta \kappa)^{2}$, where $A_{\mu \nu}$ are the random harmonic amplitudes associated with the transverse wave vectors $\boldsymbol{\kappa}_{\mu \nu}, k$ is the propagating field wave number, $\Delta z$ is the thickness of the phase screen along the propagation path, $\Phi_{n}\left(\boldsymbol{\kappa}_{\mu \nu}\right)$ is the spectral power density of the refractive index fluctuations, taken to be the Kolmogorov power-law model [see Eq. (2), later], and $\Delta \kappa$ is the grid spacing in the transverse frequency domain. By applying the Fourier transform to the random amplitudes $A_{\mu \nu}$, we generate the random phase fluctuations $\Delta \theta_{l m}$ over a $N \times N$ grid. This procedure is repeated at each phase screen along the path.

We choose the simple Kolmogorov spectrum model for our analysis because it is consistent with that used in related studies and also because we are interested primarily in first-order atmospheric effects, not necessarily the additional subtleties associated with the presence of inner scale and outer scale. However, the lowest part of the spectrum [Eq. (2)] approaching the singularity at $\kappa=0$ is not properly sampled with typical grid values. To overcome this problem, we have made use of the subharmonics technique, ${ }^{9,10}$ using a correction term to the power spectrum in Eq. (2) at the subharmonic range to compensate for the power losses due to undersampling. ${ }^{11}$

To stay within the limitations of weak irradiance fluctuations, the maximum optical path length between transmitter and receiver is taken to be $10 \mathrm{~km}$ at high altitude so that the refractive index structure constant is on the order of $C_{n}^{2}=1.39 \times 10^{-16} \mathrm{~m}^{-2 / 3}$. The free-space wavelength of the optical beam is $\lambda=1.6 \mu \mathrm{m}$, and the beam radius at the transmitter is generally taken to be either 10 or $20 \mathrm{~cm}$. The turbulent optical path is simulated by placing ten phase screens equally spaced at $1-\mathrm{km}$ increments. Each phase screen consists of $512 \times 512$ grid points, with a total screen size of $0.6 \mathrm{~m}$ for the $10-\mathrm{cm}$ beam and $1 \mathrm{~m}$ for the $20-\mathrm{cm}$ beam. The numerical results plotted in the figures correspond to mean values obtained by performing 8,000 realizations.

We define the hot spot as the "point" within the beam profile of maximum irradiance. In the simulation runs, this can correspond to either a single pixel or a small area that contains the maximum power. Because of difficulties in sometimes identifying a single pixel of maximum irradiance, we based our calculations on a small circular window area of radius $0.5 W(z)$, where $W(z)$ is the free-space spot radius at distance $z$. However, the calculated mean-square location of the hot spot with respect to the boresight or centroid is dependent on the size of the small window area chosen to define the hot spot. In general, the predicted displacements decrease with increasing window area size.

\section{Theoretical Models for Beam Wander}

Some of the theoretical models we use for describing various beam wander phenomena have been developed in related papers, ${ }^{8,12,13}$ and so their derivation will not be repeated here. In our work, we identify separate models for centroid displacement and hot spot displacement, the latter of which is a multiple of the former.

\subsection{Centroid Displacement}

Fante ${ }^{14}$ was one of the first scientists to relate the centroid displacement to the long-term and short-term spot sizes, the latter defined by the reduction in irradiance by a factor of $1 / e$. Specifically, if we let $a_{0}$ denote the spot radius of the beam at the transmitter based on the $1 / e$ point of the irradiance, then the long-term spot radius of the beam $a_{\mathrm{LT}}$ is related to the mean-square centroid displacement $\left\langle\beta_{c}^{2}\right\rangle$ and the short-term beam radius $a_{\mathrm{ST}}$ by $a_{\mathrm{LT}}^{2}=a_{\mathrm{ST}}^{2}+\left\langle\beta_{c}^{2}\right\rangle$. (Ref. 14) Various models developed for $\left\langle\beta_{c}^{2}\right\rangle$ generally have the same functional form but slightly different scaling constants. The model we use here for $\left\langle\beta_{c}^{2}\right\rangle$ is that developed Churnside and Lataitis, ${ }^{13}$ namely, $\left\langle\beta_{c}^{2}\right\rangle=0.56\left\langle r_{c}^{2}\right\rangle$, where $\left\langle r_{c}^{2}\right\rangle$ includes both centroid and hot spot displacements as defined later by Eq. (1).

\subsection{Beam Wander Variance and Spot Size}

The basic model for the relative variance of centroid and hot spot displacement is defined by $7,8,12,13$

$$
\begin{aligned}
\left\langle r_{c}^{2}\right\rangle= & 1.78\left\langle\beta_{c}^{2}\right\rangle=7.25 L^{2} W_{0}^{-1 / 3} \\
& \times \int_{0}^{L} C_{n}^{2}(z)(1-z / L)^{2}\left|1-z / F_{0}\right|^{-1 / 3} \mathrm{~d} z,
\end{aligned}
$$

where $C_{n}^{2}(z)$ is the structure parameter of the refractive index as a function of distance $z, L$ is the total propagation path length, $W_{0}=\sqrt{2} a_{0}$ is the spot radius of the beam in the plane of the transmitter based on the reduction in irradiance by a factor of $1 / e^{2}, F_{0}$ is the corresponding geometric focus or phase front radius of curvature, and the bracket \langle\rangle denotes an ensemble average. The result (1) is based on the Kolmogorov power-law spectrum

$\Phi_{n}(\kappa, z)=0.033 C_{n}^{2}(z) \kappa^{-11 / 3}$,

which, for constant $C_{n}^{2}$, reduces to

$\left\langle r_{c}^{2}\right\rangle=\left\{\begin{array}{cl}2.42 C_{n}^{2} L^{3} W_{0}^{-1 / 3}{ }_{2} F_{1}\left(\frac{1}{3}, 1 ; 4 ; \frac{L}{F_{0}}\right), & \frac{L}{F_{0}} \leqslant 1 \\ 0.54 C_{n}^{2} L^{3} W_{0}^{-1 / 3}\left(\frac{F_{0}}{L}\right)^{3}\left[9+\frac{4 L}{F_{0}}\left(\frac{5 L}{F_{0}}-6\right)+9\left(\frac{L}{F_{0}}-1\right)^{8 / 3}\right], & \frac{L}{F_{0}}>1 .\end{array}\right.$ 
The function ${ }_{2} F_{1}$ is the hypergeometric function of Gauss, which equals unity in the case of a collimated beam $\left(F_{0}\right.$ $=\infty)$ and 1.13 in the case of a focused beam $\left(F_{0}=L\right)$. The second expression in Eq. (3), which we believe is a new result, is applicable for the case of a beam focused in front of the receiver.

Following Fante, ${ }^{14}$ we write the beam wander variance (1) as

$\left\langle r_{c}^{2}\right\rangle=W_{\mathrm{LT}}^{2}-W_{\mathrm{ST}}^{2}$,

where $W_{\mathrm{LT}}$ and $W_{\mathrm{ST}}$ denote the long-term and short-term spot radii, respectively. In fact, we use Eq. (4) to define the short-term beam radius [see Eq. (7), below]. The long-term spot radius deduced from conventional Rytov theory leads to 7

$W_{\mathrm{LT}}=W\left[1+1.33 \sigma_{R}^{2}\left(\frac{2 L}{k W^{2}}\right)^{5 / 6}\right]^{1 / 2}$,

where $\sigma_{R}^{2}=1.23 C_{n}^{2} k^{7 / 6} L^{11 / 6}$ is the Rytov variance, $k=2 \pi / \lambda$ is the optical wave number, $\lambda$ is the wavelength, and $W$ is the free-space spot radius in the plane of the receiver, i.e.,

$W=W_{0}\left[\left(1-\frac{L}{F_{0}}\right)^{2}+\left(\frac{2 L}{k W_{0}^{2}}\right)^{2}\right]^{1 / 2}$.

Based on Eq. (4), the short-term spot radius $W_{\mathrm{ST}}$ is defined by

$W_{\mathrm{ST}}=\left(W_{\mathrm{LT}}^{2}-\left\langle r_{c}^{2}\right\rangle\right)^{1 / 2}$.

\subsection{Hot Spot Displacement}

The hot spot displacement from the centroid is a result of the short-term beam being skewed from a Gaussian profile. For a collimated beam, the mean-square displacement of the hot spot from the boresight $\sigma_{0}^{2}$ and that from the beam centroid $\sigma_{c}^{2}$ can be estimated, respectively, from the empirical relations 8

$\sigma_{0}^{2} \approx W_{\mathrm{LT}}^{2}-W^{2}$

$\sigma_{c}^{2} \approx W_{\mathrm{ST}}^{2}-W^{2}$

Moreover, from Eq. (4) and these last expressions, it follows that

$\left\langle r_{c}^{2}\right\rangle \approx \sigma_{0}^{2}-\sigma_{c}^{2}$.

That is, the beam wander variance defined by Eq. (1) can be interpreted as an estimate of the difference in meansquare displacements of the hot spot from the boresight and from the centroid.

\section{Scintillation Theory}

Several publications during the last few years indicate a renewed interest in studying the wander-induced scintillation caused by beam wander along various horizontal and uplink propagation paths. ${ }^{1,12,15-17}$ Estimates of the induced scintillation from beam wander were given in Baker and Benson, ${ }^{17}$ and a similar study by Andrews et al. ${ }^{12}$ led to a different analytic expression for the wander-induced scintillation associated with an uplink path to space.

The derivation of the scintillation expressions given in this section have been published elsewhere, ${ }^{7,12}$ so we will simply summarize the theoretical models rather than derive them again. The reader interested in the details can consult the references.

\subsection{Beam Parameters}

We assume that the spot size radius and phase front radius of curvature at the transmitter of the propagating beam are $W_{0}$ and $F_{0}$, respectively. We use the convention that $F_{0}$ $>0$ for a convergent beam and $F_{0}<0$ for a divergent beam. For a propagation path of length $L$ along the positive $z$ axis, it is convenient to describe free-space propagation by use of two sets of nondimensional beam parameters ${ }^{7}$

$$
\begin{aligned}
& \Theta_{0}=1-\frac{L}{F_{0}}, \quad \Lambda_{0}=\frac{2 L}{k W_{0}^{2}}, \\
& \Theta=1+\frac{L}{F}=\frac{\Theta_{0}}{\Theta_{0}^{2}+\Lambda_{0}^{2}}, \quad \Lambda=\frac{2 L}{k W^{2}}=\frac{\Lambda_{0}}{\Theta_{0}^{2}+\Lambda_{0}^{2}} .
\end{aligned}
$$

The first set of beam parameters (11) characterizes the beam at the transmitter, and the second set (12) characterizes the beam at the receiver, where $W$ and $F$ represent the spot-size radius and phase front radius of curvature of the beam as viewed in the receiver plane. In some cases, we also use the notation $\bar{\Theta}=1-\Theta=-L / F$.

\subsection{Boundary of the $D_{1}$ Region}

The $D_{1}$ region is associated with a portion of the propagation path for a beam wave where the Rytov perturbation method is not appropriate. Baker ${ }^{5}$ recently defined the borders of the $D_{1}$ region by a set of Fresnel numbers satisfying

$N_{L}=\frac{\left|\Theta_{0}\right|}{\Lambda_{0}}=\frac{\left|1-L / F_{0}\right|}{\Lambda_{0}}<1, \quad N_{\tau}=\frac{L}{z_{\tau}} \frac{\left|1-z_{\tau} / F_{0}\right|}{\Lambda_{0}}>1$,

where $z_{\tau}$ is the effective range of the turbulence. Using the first Fresnel inequality, it follows that the $D_{1}$ region for a convergent beam includes the regime where the free-space longitudinal phase shift ${ }^{7}$ satisfies

$\varphi=\tan ^{-1} \frac{\Lambda_{0}}{\Theta_{0}}>\frac{\pi}{4}$.

In fact, if we include the geometric focus, the $D_{1}$ region covers that portion of the propagation path for which $\pi / 4$ $<\varphi<3 \pi / 4$, with $\varphi=\pi / 2$ identifying the propagation distance to the geometric focus. Depending on beam size and location of geometric focus, we can use the longitudinal phase (14) to estimate the propagation range over which the $D_{1}$ region occurs.

\subsection{Untracked Beam}

Under the Rytov theory, the scintillation index of a Gaussian-beam wave takes the form 


$$
\begin{aligned}
\sigma_{I}^{2}(r, L)= & 8 \pi^{2} k^{2} L \int_{0}^{1} \int_{0}^{\infty} \kappa \Phi_{n}(\kappa, z) \exp \left(-\frac{\Lambda L \kappa^{2} \xi^{2}}{k}\right) \\
& \times\left\{I_{0}(2 \Lambda r \kappa \xi)-\cos \left[\frac{L \kappa^{2}}{k} \xi(1-\bar{\Theta} \xi)\right]\right\} \mathrm{d} \kappa \mathrm{d} \xi
\end{aligned}
$$

where $I_{0}(x)$ is a modified Bessel function of the first kind, and $\xi=1-z / L$ is a normalized distance variable. If we insert the Kolmogorov spectrum (2) with constant $C_{n}^{2}$ into the integrals in (15), the resulting scintillation index becomes

$$
\begin{aligned}
\sigma_{I}^{2}(r, L)= & 3.86 \sigma_{R}^{2} \operatorname{Re}\left[i_{2}^{5 / 6} F_{1}\left(-\frac{5}{6}, \frac{11}{6} ; \frac{17}{6} ; 1-\Theta+i \Lambda\right)\right] \\
& -2.64 \sigma_{R}^{2} \Lambda^{5 / 6}{ }_{1} F_{1}\left(-\frac{5}{6} ; 1 ; \frac{2 r^{2}}{W^{2}}\right),
\end{aligned}
$$

where Re denotes the real part of the argument, and ${ }_{1} F_{1}$ is the confluent hypergeometric function. In the presence of beam wander, Andrews et al. ${ }^{7,12}$ have discussed the notion of an effective pointing error $\sigma_{p e}$ that arises from a subset of the random scale sizes that define the beam wander variance $\left\langle r_{c}^{2}\right\rangle$. In the case of an untracked beam, this subset of scale sizes, bounded from above by the atmospheric coherence width $r_{0}$, yields an on-axis value of the scintillation index described by

$$
\begin{aligned}
\sigma_{I}^{2}\left(\sigma_{p e}, L\right)_{\text {untracked }}= & 3.86 \sigma_{R}^{2} \operatorname{Re}\left[i ^ { 5 / 6 } { } _ { 2 } F _ { 1 } \left(-\frac{5}{6}, \frac{11}{6} ; \frac{17}{6} ; 1-\Theta\right.\right. \\
& +i \Lambda)]-2.64 \sigma_{R}^{2} \Lambda^{5 / 6}{ }_{1} F_{1}\left(-\frac{5}{6} ; 1 ; \frac{2 \sigma_{p e}^{2}}{W^{2}}\right),
\end{aligned}
$$

where the mean-square pointing error for a focused beam is

$$
\sigma_{p e}^{2}=\left\langle r_{c}^{2}\right\rangle\left[1-\frac{8}{9}\left(\frac{C_{r}^{2} W_{0}^{2} / r_{0}^{2}}{1+0.5 C_{r}^{2} W_{0}^{2} / r_{0}^{2}}\right)^{1 / 6}\right] \text {. }
$$

The quantity $r_{0}=\left(0.16 C_{n}^{2} k^{2} L\right)^{-3 / 5}$ is the atmospheric coherence width (i.e., Fried's parameter) of a reciprocal propagating point source from the receiver at distance $L$, and the parameter $C_{r}$ is a scaling constant on the order of $2 \pi$ but its exact value has not yet been determined. For an uplink beam to space, the value $C_{r}=\pi$ was used, ${ }^{12}$ whereas $C_{r}$ $=1.5 \pi$ in the present study. The difference in value for the scaling constant is attributed mostly to the fact that $r_{0}$ reduces to the plane wave atmospheric coherence width in the uplink beam case. Last, although not strictly correct, we will also use Eq. (18) in the following analysis to estimate the pointing error that arises from a beam focused at some intermediate plane along the path other than at the receiver plane.

\subsection{Tracked Beam}

A common method of tracking a beam involves removal of the tilt component from the wandering beam. Theoretically, the rms tilt from the far-field beam can be removed using the two-axis Zernike tilt variance ${ }^{12,18}$
$T_{z}^{2}=0.32\left(\frac{\lambda}{2 W_{0}}\right)^{2}\left(\frac{2 W_{0}}{r_{0}}\right)^{5 / 3}$

By subtracting the rms tilt displacement $T_{z} L$ from the rms beam wander, we obtain the tilt-corrected pointing error variances

$$
\sigma_{p e, T C}^{2}=\left[\left(\left\langle r_{c}^{2}\right\rangle\right)^{1 / 2}-T_{z} L\right]^{2}\left[1-\frac{8}{9}\left(\frac{C_{r}^{2} W_{0}^{2} / r_{0}^{2}}{1+0.5 C_{r}^{2} W_{0}^{2} / r_{0}^{2}}\right)^{1 / 6}\right] .
$$

In this case, the on-axis scintillation index for a tiltcorrected beam becomes

$$
\begin{aligned}
\sigma_{I}^{2}\left(\sigma_{p e, T C}, L\right)_{\text {tracked }}= & 3.86 \sigma_{R}^{2} \operatorname{Re}\left[i^{5 / 6}{ }_{2} F_{1}\right. \\
& \left.\times\left(-\frac{5}{6}, \frac{11}{6} ; \frac{17}{6} ; 1-\Theta+i \Lambda\right)\right] \\
& -2.64 \sigma_{R}^{2} \Lambda^{5 / 6}{ }_{1} F_{1}\left(-\frac{5}{6} ; 1 ; \frac{2 \sigma_{p e, T C}^{2}}{W^{2}}\right) .
\end{aligned}
$$

Note that Eq. (21) has the same form as Eq. (17) for an untracked beam, but with the wander-induced pointing error $\sigma_{p e}$ replaced by the tilt-corrected pointing error $\sigma_{p e, T C}$. However, because the beam wander variance (3) is generally a little larger than the tilt variance (19), the tiltcorrected beam has a residual pointing error term that may contribute a nonzero amount to the detected on-axis scintillation index.

\section{Comparison of Results}

In this section, we show a variety of simulation results and compare them with the corresponding theoretical results provided in Secs. 3 and 4. To study the onset of beam wander effects, all simulation results are based on the assumption of a convergent beam propagating along a $10-\mathrm{km}$ horizontal path at high altitude for which weak fluctuation theory is applicable. The Kolmogorov power-law spectrum (2) with structure parameter $C_{n}^{2}=1.39 \times 10^{-16} \mathrm{~m}^{-2 / 3}$ is used in all simulation and theoretical results.

Because the following analysis involves two types of convergent Gaussian beam, we will designate these two beam types as beam $\mathrm{F}_{1}$ and beam $\mathrm{F}_{2}$, where

- Beam $\mathrm{F}_{1}$ : Beam focused at the end of a $10-\mathrm{km}$ path $\left(F_{0}=10 \mathrm{~km}\right)$

- Beam $\mathrm{F}_{2}$ : Beam focused at the midpoint of a $10-\mathrm{km}$ path $\left(F_{0}=5 \mathrm{~km}\right)$

\subsection{Rms Beam Centroid Displacement}

Shown in Fig. 1 for beam $\mathrm{F}_{1}$ are the simulation and theoretical results for the beam centroid displacement of three beams with transmitter radii of $5 \mathrm{~cm}, 10 \mathrm{~cm}$, and $50 \mathrm{~cm}$. In this case, we have chosen three beam sizes to better illustrate how the size of the beam affects beam wander. Theoretical curves in the figure are calculated from the expression $\left(\left\langle\beta_{c}^{2}\right\rangle\right)^{1 / 2}=\left(0.56\left\langle r_{c}^{2}\right\rangle\right)^{1 / 2}$. The theoretical and simulation 


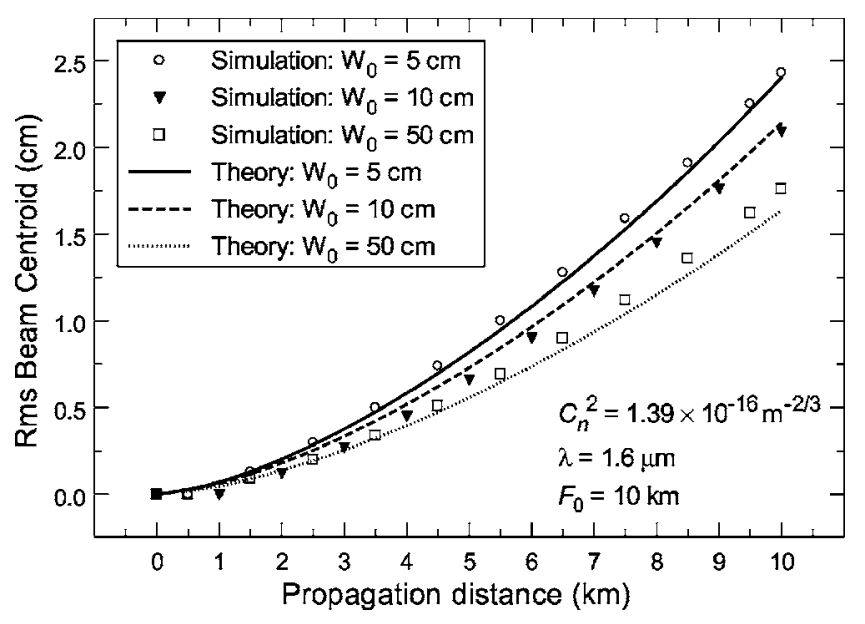

Fig. 1 Rms centroid movement from boresight for beam $F_{1}$ as a function of propagation distance. Theoretical results are based on $\left(\left\langle\beta_{c}^{2}\right\rangle\right)^{1 / 2}=\left(0.56\left\langle r_{c}^{2}\right\rangle\right)^{1 / 2}$.

results are in closer agreement for the smaller beam sizes, but still within $7 \%$ for the $50-\mathrm{cm}$ beam at $10 \mathrm{~km}$. In Fig. 2, we show centroid wander results for beam $\mathrm{F}_{2}$ with transmitter radii of $10 \mathrm{~cm}$ and $20 \mathrm{~cm}$. In this case, the theoretical and simulation results are in good agreement for both illustrated beam sizes.

\subsection{Kurtosis Excess}

In the case of a collimated beam along the $10-\mathrm{km}$ path, the long-term and short-term mean irradiance profiles will be nonskewed and maintain a near-Gaussian shape along the whole propagation path. The situation is different for a focused beam, however, where the mean irradiance profile is nonskewed but may still differ from Gaussian shape along the propagation path near the geometric focus, thus requiring an additional parameter to account for the deviation from Gaussian. Usually, kurtosis plays this role. In our case, we have considered the kurtosis excess coefficient, which is defined by

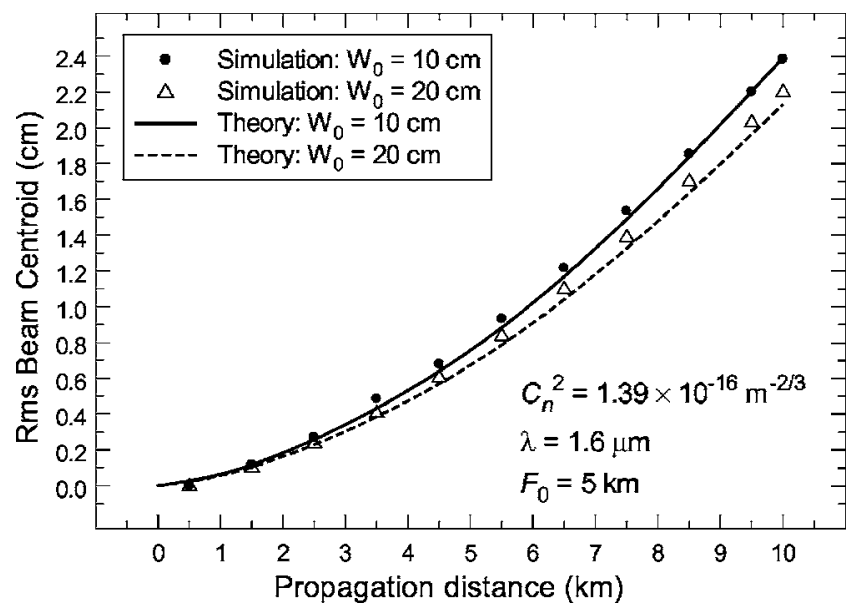

Fig. 2 Same as Fig. 1 for beam $F_{2}$.

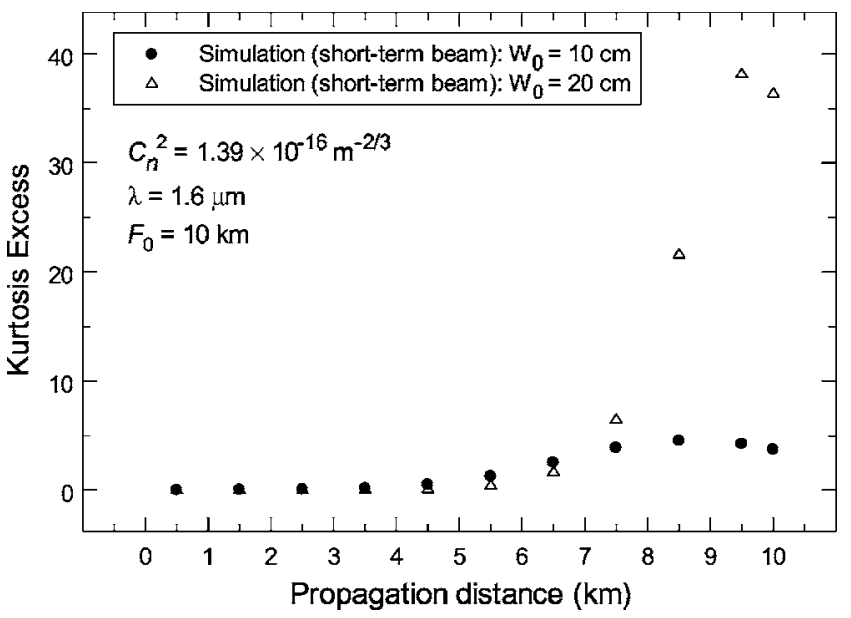

Fig. 3 Coefficient of kurtosis excess for beam $F_{1}$ calculated from Eq. (22) for the short-term beam and plotted as a function of propagation distance.

$\left\langle\gamma_{2}\right\rangle \equiv\left\langle\frac{\mu_{4}}{\sigma^{4}}\right\rangle-3$,

where $\mu_{4}$ is the fourth central moment of the irradiance distribution, taken along either the $x$ axis or the $y$ axis. The kurtosis excess vanishes for a Gaussian profile. A positive kurtosis excess value indicates that the irradiance profile is either "peaked" more than a Gaussian profile or has "flatter" tails. A negative value indicates that the beam profile is more flattened.

Simulation results for the kurtosis excess of the averaged short-term beam are shown in Figs. 3 and 4. In Fig. 4, we also include the kurtosis excess for the long-term $10-\mathrm{cm}$ beam, which is slightly lower than that of the short-term beam. It is interesting to note that the kurtosis excess may reach high positive values near the geometric focus, especially for the $20-\mathrm{cm}$ beam. It is also a remarkable fact that once beam $\mathrm{F}_{2}$ passes through the geometric focus at $5 \mathrm{~km}$, the mean irradiance profile returns back to near-Gaussian, i.e., the kurtosis excess tends to zero once again

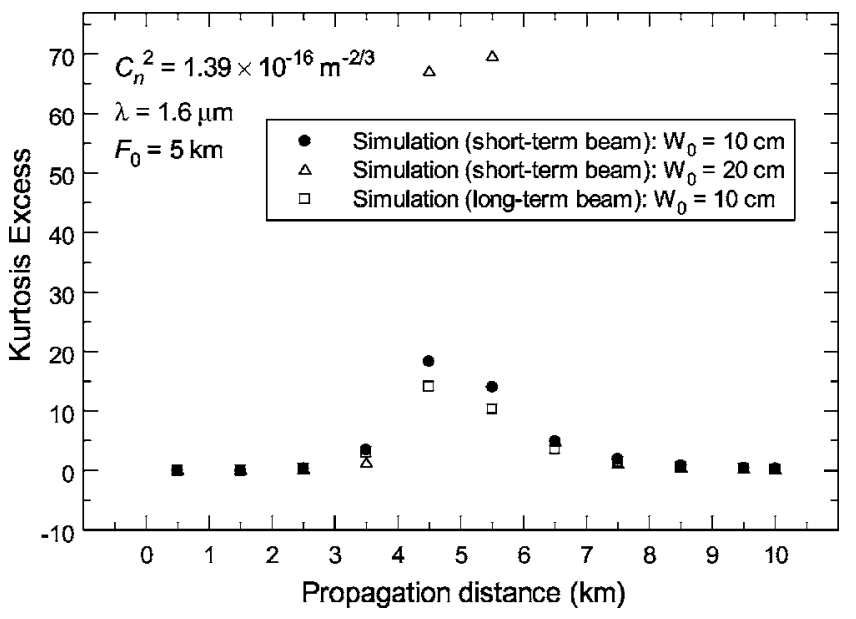

Fig. 4 Same as Fig. 3 for beam $F_{2}$. Also shown here is the longterm kurtosis excess for the $10-\mathrm{cm}$ beam. 


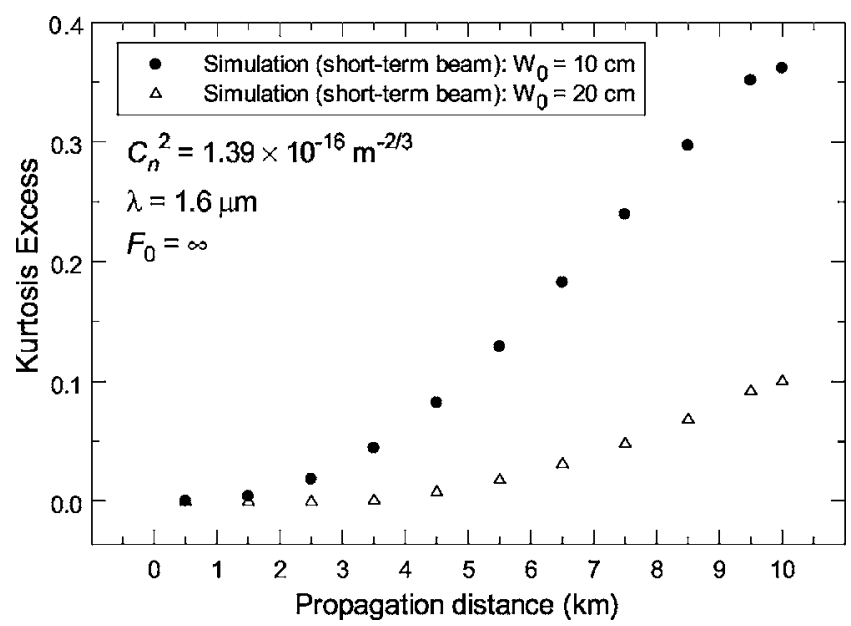

Fig. 5 Same as Fig. 3 for a collimated beam.

(see Fig. 4). Last, the portion of the propagation path between $4.7 \mathrm{~km}$ and $5.3 \mathrm{~km}$ corresponds roughly with the longitudinal phase shift $\pi / 4<\varphi<3 \pi / 4$ for the $20-\mathrm{cm}$ beam.

For contrast, we show in Fig. 5 the kurtosis excess for two collimated beams of sizes similar to the convergent beam cases. The kurtosis excess is much less than unity for both beam sizes in this case, indicating a near-Gaussian profile shape over the entire propagation path.

\subsection{Beam Radius}

We tested two different methods to obtain numerical values for the long-term spot size. The first method is based on the property that the $1 / e^{2}$ irradiance radius of a Gaussian beam can be expressed as $2 \sigma$, where $\sigma$ is the standard deviation deduced from the first and second moments of the irradiance. The second method is based on associating the spotsize radius with a circle that contains $86.47 \%$ of the total beam energy.

In Fig. 6, we present simulation results for beam $F_{1}$ based on both methods for the long-term spot size of a

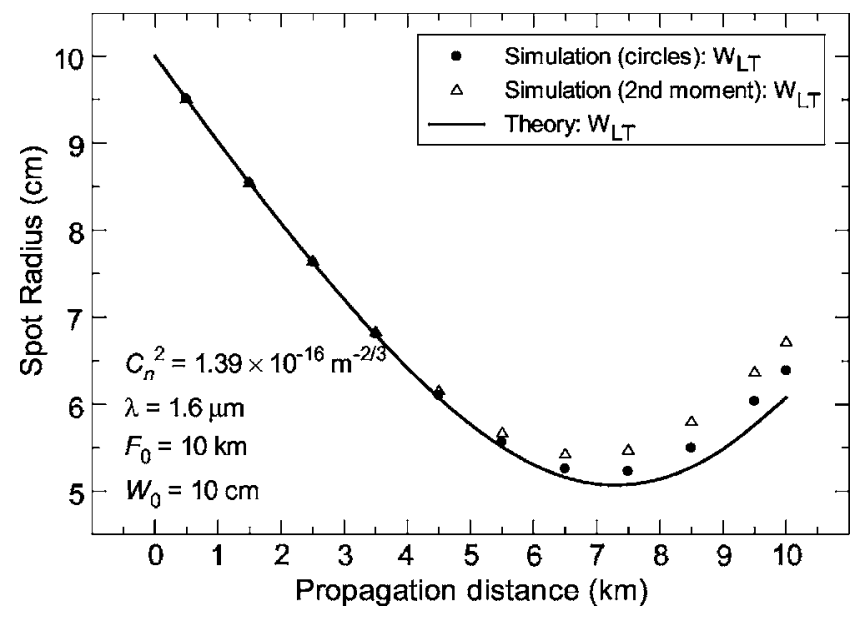

Fig. 6 Long-term beam radius of beam $F_{1}$ as a function of propagation distance. Two methods of calculating spot size are featured in the simulation results. Theoretical results are based on Eq. (5). 10-cm beam plotted as a function of propagation distance. We found that the values obtained from the standard deviation method were generally higher than those obtained from the $86.47 \%$ energy circle method near the focus, although prior to the focus, the two methods produced quite similar results. A theoretical curve based on Eq. (5) is also shown in Fig. 6. In general, the theoretical estimate of the longterm spot radius of the beam is in good agreement with the simulation results. The largest discrepancy occurs at $10 \mathrm{~km}$, but the theoretical result is still within $5 \%$ of the value for the simulation point. The discrepancies between these two methods and with the theoretical estimates predicted from Rytov theory can be explained by the fact that in the vicinity of the focus, the beam profiles differ from being pure Gaussian, as indicated by the high kurtosis excess values obtained in Sec. 5.2 (see Figs. 3 and 4). This issue will be treated in greater detail in the following subsection.

\subsection{Mean Irradiance Profile}

For the long-term beam, the mean irradiance profile is often approximated by the Gaussian function

$\langle I(r, L)\rangle \approx \frac{W_{0}^{2}}{W_{\mathrm{LT}}^{2}} \exp \left(-\frac{2 r^{2}}{W_{\mathrm{LT}}^{2}}\right)$,

where the long-term spot radius is defined by Eq. (5). Except in the vicinity of the geometric focus of a convergent beam, where the kurtosis excess shown in Figs. 3 and 4 begins to greatly increase, we expect the Gaussian approximation (23) to be quite close to the exact mean irradiance profile predicted by Rytov theory, namely, ${ }^{7}$

$$
\begin{aligned}
\langle I(r, L)\rangle= & \frac{W_{0}^{2}}{W^{2}} \exp \left(-\frac{2 r^{2}}{W^{2}}\right) \\
& \times \exp \left[-1.33 \sigma_{R}^{2} \Lambda_{1}^{5 / 6} F_{1}\left(-\frac{5}{6} ; 1 ; \frac{2 r^{2}}{W^{2}}\right)\right] .
\end{aligned}
$$

Of course, the validity of Eq. (24) requires $\sigma_{R}^{2} \Lambda^{5 / 6} \ll 1$, ensuring the conditions of weak fluctuation theory.

We show simulation results for the long-term beam irradiance profile (scaled to a maximum mean irradiance of unity) on a logarithmic scale in Fig. 7 , for the $20-\mathrm{cm} \mathrm{F}_{2}$ beam at distance $4.5 \mathrm{~km}$ from the transmitter. To compare the accuracy of the Gaussian approximation at this distance, we also plot the Gaussian function (23) using the spot radius obtained from the $86.47 \%$ energy circle as well as that from the second central moment, each scaled by $W_{0}^{2} / W_{\mathrm{LT}}^{2}$ for normalization of the profile to a maximum value of unity. Note that the circle method predicts a spot size that matches the simulation results more closely in the center part of the beam, but neither Gaussian approximation reproduces the behavior of the simulation results at the tailgates, as indicated by a large positive kurtosis excess (recall Fig. 4).

To view the transition of the mean irradiance profile from a Gaussian shape to a non-Gaussian shape as the beam approaches the geometric focus, we display scaled simulation results for the $20-\mathrm{cm} \quad \mathrm{F}_{2}$ long-term beam in Fig. 8 at distances of $3.5 \mathrm{~km}, 4.5 \mathrm{~km}$, and $5 \mathrm{~km}$ from the source. It is interesting that the scaled simulation results resemble a Gaussian shape at distance $3.5 \mathrm{~km}$ but exhibit high tails in 


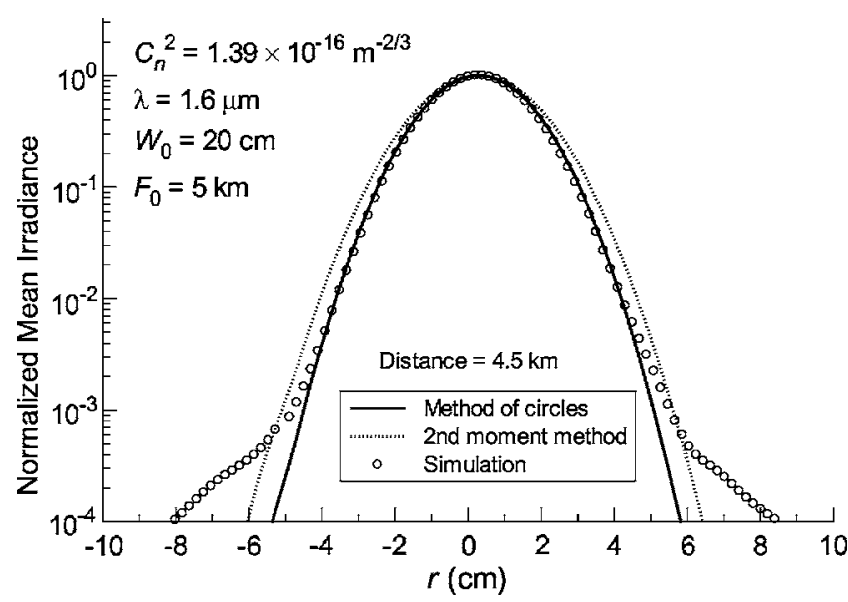

Fig. 7 Mean irradiance profile of the $20-\mathrm{cm}$ beam $F_{2}$ at $4.5 \mathrm{~km}$. The Gaussian approximation (23) is scaled by the factor $W_{0}^{2} / W_{1 T}^{2}$, and the simulation results are scaled so that the maximum value is unity.

the profile at distances between $4.5 \mathrm{~km}$ and the geometric focus, which is consistent with the kurtosis values obtained in Fig. 4. Nevertheless, the region near the peak that carries most of the energy of the beam can still be approached by Eq. (23), provided that the $86.47 \%$ energy circle is used to estimate the value of $W_{\mathrm{LT}}$.

We have also compared in Fig. 9 the long-term beam profiles obtained from simulation and scaled to a maximum value of unity, with the mean irradiance profiles predicted by Eq. (24), scaled by $W_{0}^{2} / W_{\mathrm{LT}}^{2}$, for the same beam of Figs. 7 and 8 , at distances of $4.5 \mathrm{~km}, 5 \mathrm{~km}$, and $5.5 \mathrm{~km}$. The Rytov model matches the simulation results out to the beam radius and somewhat beyond at $4.5 \mathrm{~km}$ and again at $5.5 \mathrm{~km}$, but clearly not so around $5 \mathrm{~km}$ (consistent with the longitudinal phase shift $\pi / 4<\varphi<3 \pi / 4$ corresponding to the $D_{1}$ region). Deviations of Eq. (24) with respect to the simulation results in Fig. 9(b) arise because the Rytov approximation is not valid for this beam owing to off-axis irradiance fluctuations exceeding the limitation of weak fluctuation theory. Interestingly enough, we show in Sec. 5.6 that the on-axis scintillation values are still within the limits of weak fluctuation theory.

\subsection{Hot Spot Movement}

In Figs. 10 and 11, we plot the quantity $\left(\sigma_{0}^{2}-\sigma_{c}^{2}\right)^{1 / 2}$ for beams $F_{1}$ and $F_{2}$ with beam radii of $10 \mathrm{~cm}$ and $20 \mathrm{~cm}$ at the transmitter, using the simulation results for the hot spot displacements based on both a single pixel and a window area. Notice that there is virtually no difference in the simulation results for the quantity $\left(\sigma_{0}^{2}-\sigma_{c}^{2}\right)^{1 / 2}$, although the individual variances $\sigma_{0}^{2}$ and $\sigma_{c}^{2}$ are quite different for the single-pixel method versus the small area method. The theoretical result in all cases is the rms beam wander term $\left(\left\langle r_{c}^{2}\right\rangle\right)^{1 / 2}$. The fit of the simulation results with the theoretical result of Eq. (10) is not as good as for the collimated beam case, ${ }^{8}$ particularly at longer distances for beam $\mathrm{F}_{1}$. In part, this is caused by the difficulty in obtaining good estimates of the mean-square hot spot displacements in the vicinity of the focus of a convergent beam. On the other

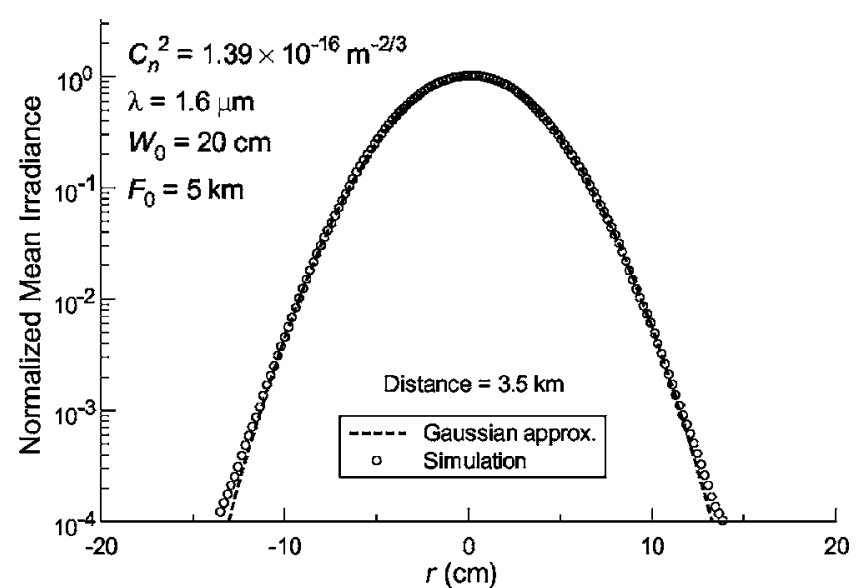

(a)

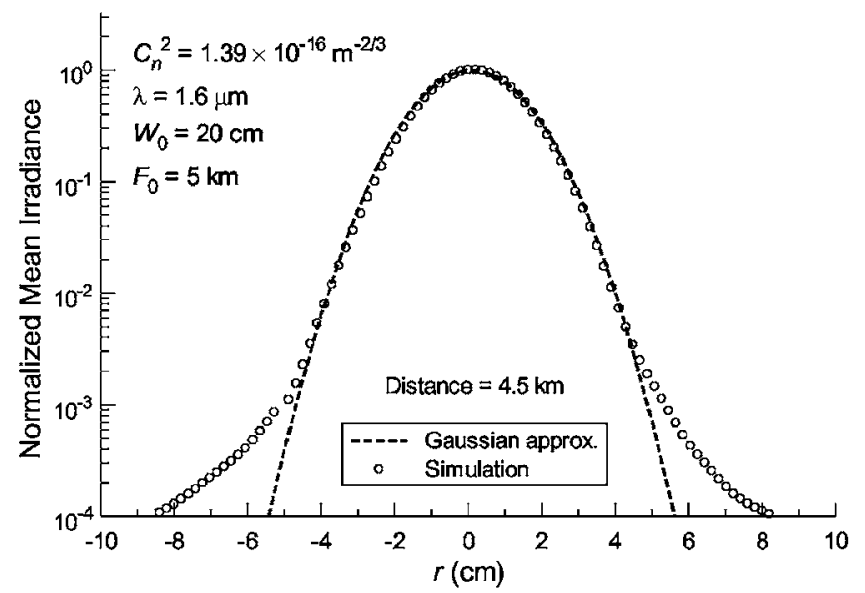

(b)

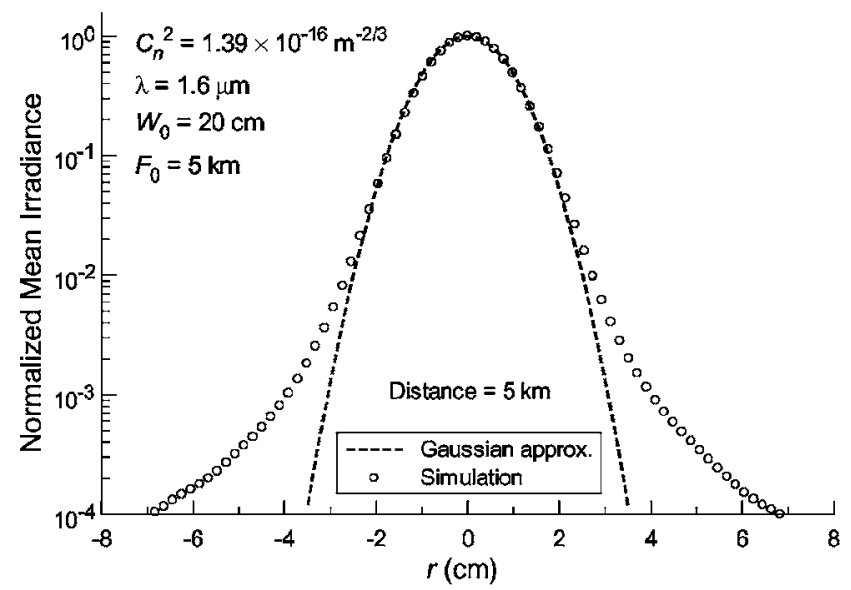

(c)

Fig. 8 Mean irradiance profile of the $20-\mathrm{cm}$ beam $\mathrm{F}_{2}$ compared with the Gaussian profile model (23) at (a) $3.5 \mathrm{~km}$, (b) $4.5 \mathrm{~km}$, and (c) $5 \mathrm{~km}$, all scaled the same as in Fig. 7.

hand, the theoretical and simulation results for beam $\mathrm{F}_{2}$ in Fig. 11(b) are in good agreement over the entire $10-\mathrm{km}$ path.

\subsection{Scintillation Index}

In Figs. 12 and 13, we show the on-axis scintillation index for beam $F_{1}$ with initial beam radii of $10 \mathrm{~cm}$ and $20 \mathrm{~cm}$ as 


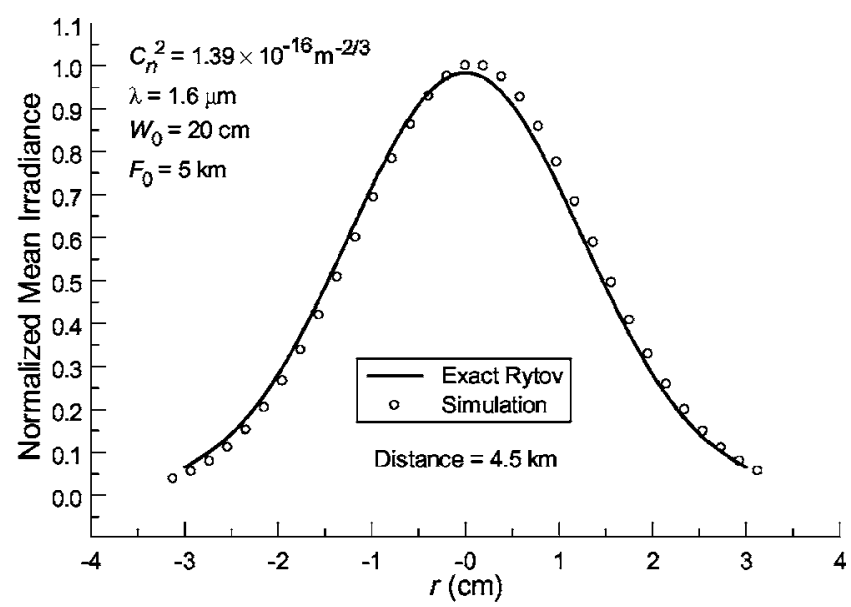

(a)

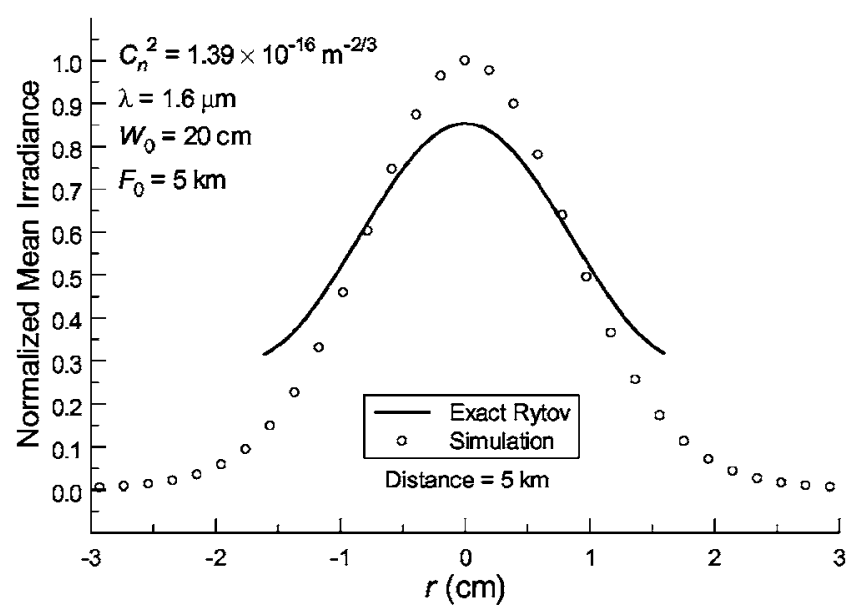

(b)

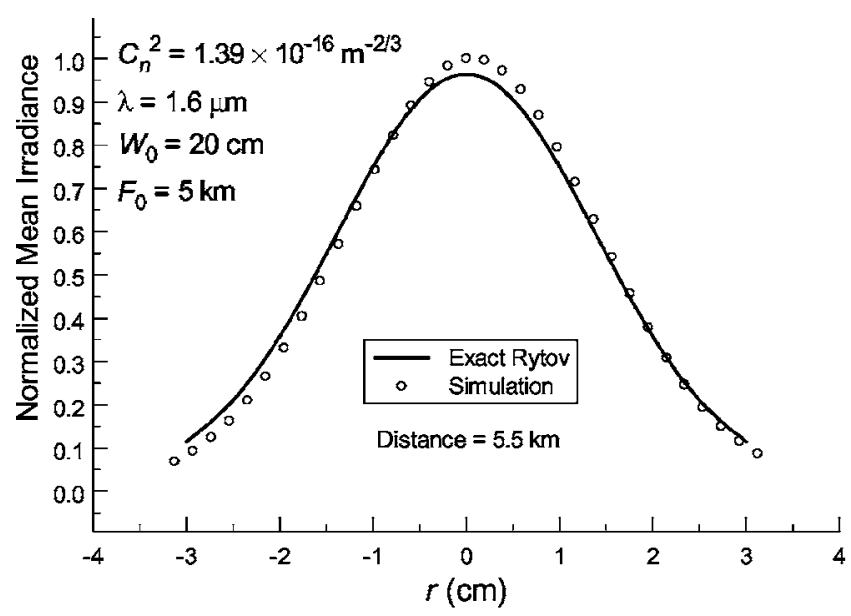

(c)

Fig. 9 Mean irradiance profile of the 20-cm beam $F_{2}$ compared with the Rytov theory (24) at (a) $4.5 \mathrm{~km}$, (b) $5 \mathrm{~km}$, and (c) $5.5 \mathrm{~km}$, all scaled the same as Fig. 7.

a function of propagation distance. The solid circles and solid curve depict the untracked beam case, and the open triangles and dashed curve portray the tracked (tilt correction) beam case. Theoretical results are based on Eqs. (17)

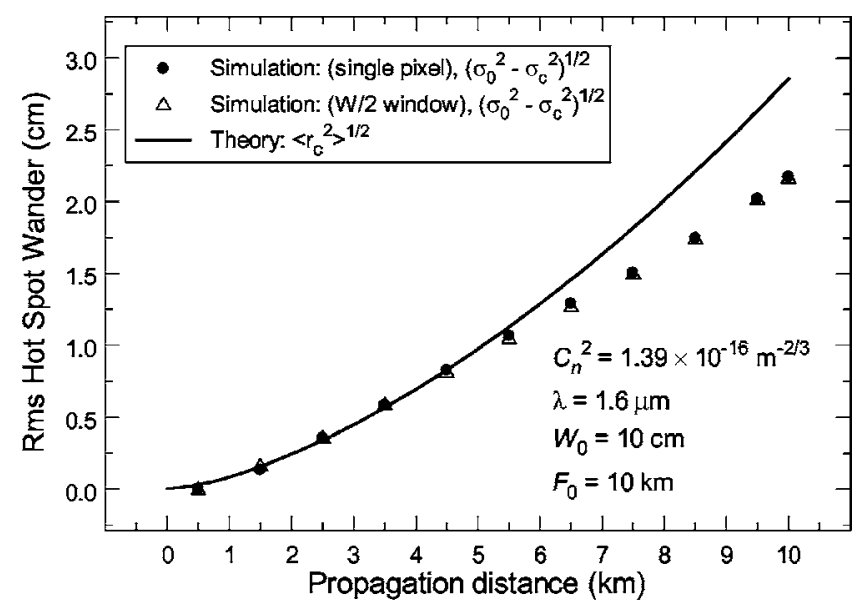

(a)

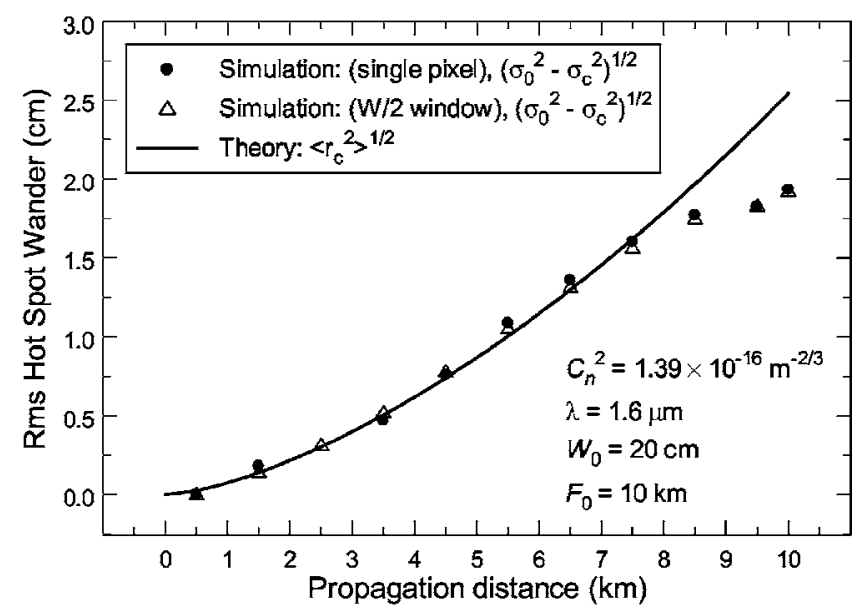

(b)

Fig. $10 \mathrm{Rms}$ beam wander of beam $F_{1}$ from the boresight as a function of propagation distance for (a) a $10-\mathrm{cm}$ beam and (b) a 20-cm beam. Theoretical results are based on Eq. (3); simulation results are based on Eq. (10).

and (21). Note that the scintillation index for the untracked and tracked beam cases begins to significantly separate around $5.5 \mathrm{~km}$ for the $10-\mathrm{cm}$ beam and around $7.5 \mathrm{~km}$ for the $20-\mathrm{cm}$ beam. These distances are close to those at which the kurtosis excess in the beam profile begins to climb (see Fig. 3), indicating the onset of the $D_{1}$ region. To illustrate the longitudinal phase shift criterion, we have marked the range in Figs. 12 and 13 at which the longitudinal phase shift is $\pi / 4$ for beam $F_{1}$. However, as already noted, the onset of the $D_{1}$ region occurs a little before the $\pi / 4$ range, where the scintillation index separates for the tracked and untracked beams. We do not expect an exact comparison of distances because the longitudinal phase shift criterion is based on free-space beam propagation whereas kurtosis excess, for example, is based on turbulence conditions. We believe that the kurtosis excess provides a better estimate for the boundary of the $D_{1}$ region.

Simulation results and theoretical results shown in Figs. 12 and 13 are generally in excellent agreement for the scintillation index in both the tracked beam case and the un- 


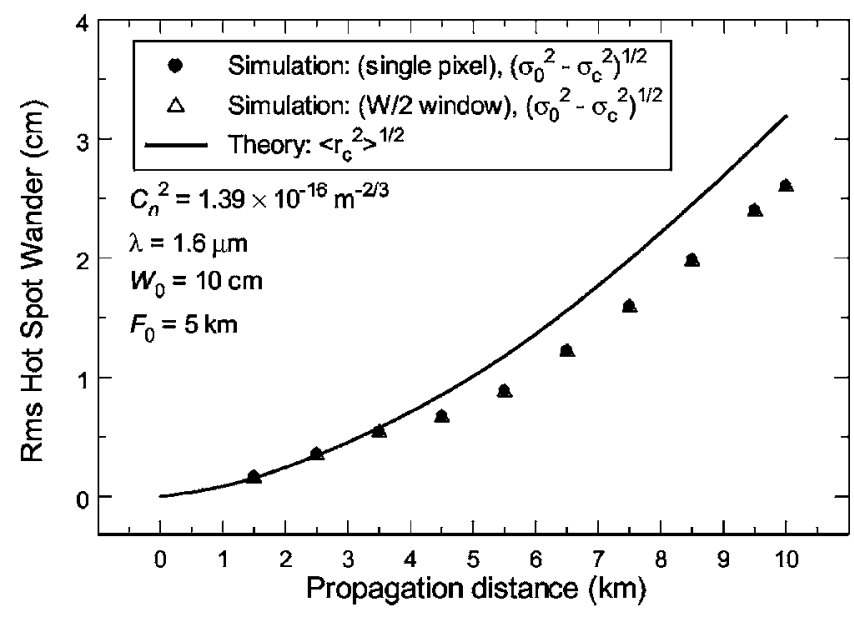

(a)

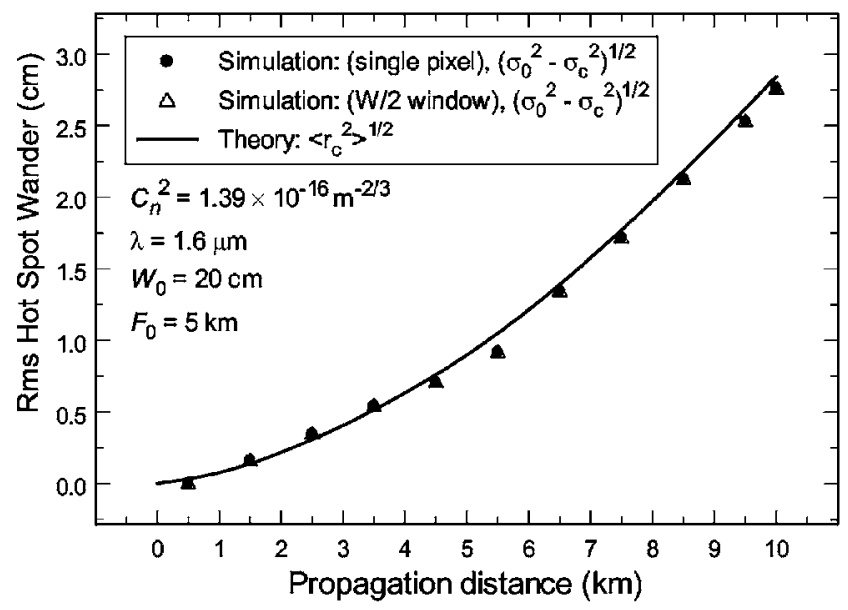

(b)

Fig. $11 \mathrm{Rms}$ beam wander of beam $\mathrm{F}_{2}$ from the boresight as a function of propagation distance for (a) a $10-\mathrm{cm}$ beam and (b) a $20-\mathrm{cm}$ beam. Theoretical results are based on Eq. (3). Simulation results are based on Eq. (10).

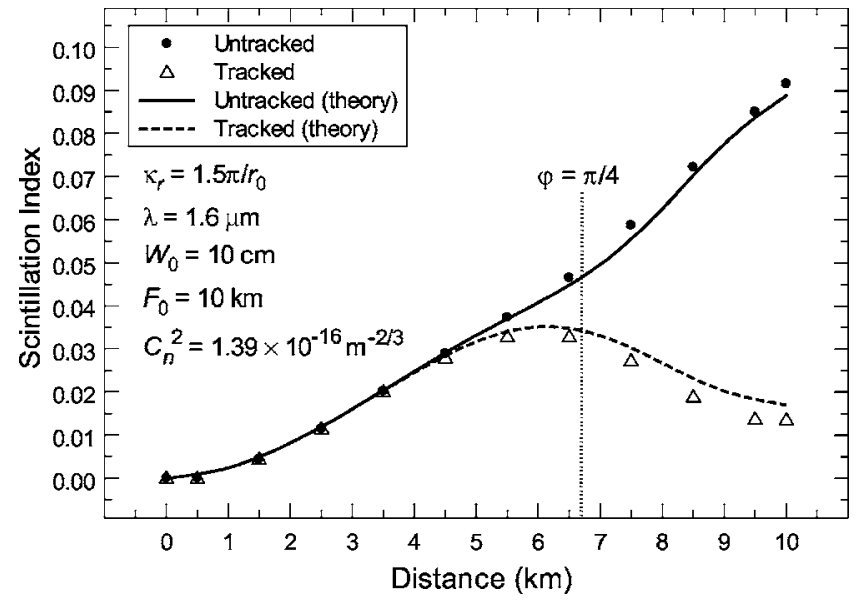

Fig. 12 On-axis scintillation index of a $10-\mathrm{cm}$ beam $F_{1}$ in both the tracked and untracked cases. The vertical dotted line corresponds to the range at which the longitudinal phase reaches $\pi / 4$.

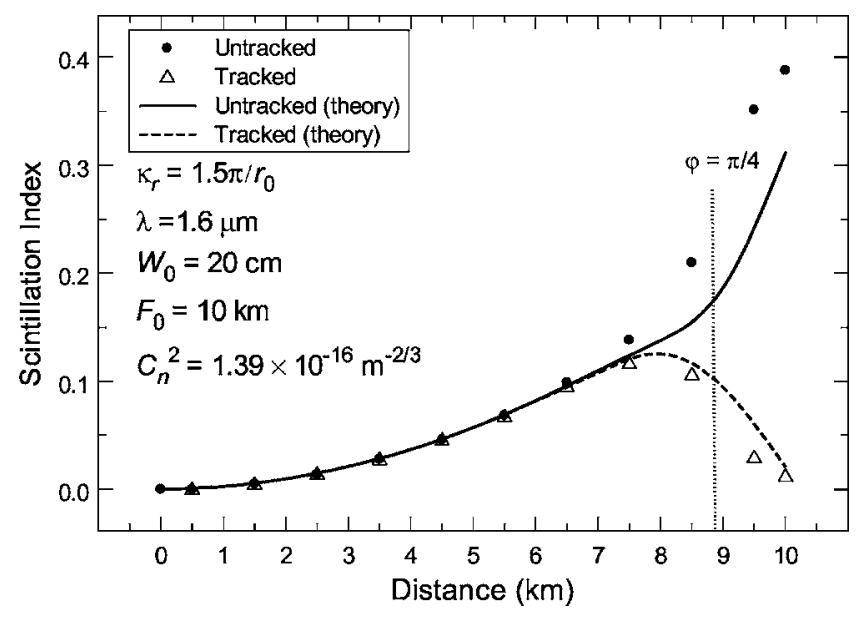

Fig. 13 Same as Fig. 12 for a $20-\mathrm{cm}$ beam.

tracked beam case. The largest difference between theory and simulation occurs between 8 and $10 \mathrm{~km}$ for the untracked 20-cm beam case (Fig. 13).

In Figs. 14 and 15, we plot the untracked and tracked scintillation index for beam $\mathrm{F}_{2}$ along the optical axis of a propagating beam. Once the results for the untracked and tracked beam cases separate in Fig. 14, they stay apart over the rest of the illustrated propagation path. However, there is a tendency for the two curves to approach each other again after $6.5 \mathrm{~km}$. In both Figs. 14 and 15, there is a dramatic change in behavior for the untracked and tracked beam cases as the beam passes through the range at which the beam is focused, corresponding to high values of kurtosis excess. The results shown in Fig. 15 are particularly interesting in that the scintillation index is nearly the same for the untracked and tracked beam cases shortly behind the geometric focus region. It is also interesting to note that the scintillation index obtained at the plane of the receiver for the untracked beam when it is focused at that plane is significantly higher than when it is focused at $5 \mathrm{~km}$. This behavior is observed both in the 10-cm (see Figs. 12 and 14) and 20-cm (see Figs. 13 and 15) beams, thus suggesting

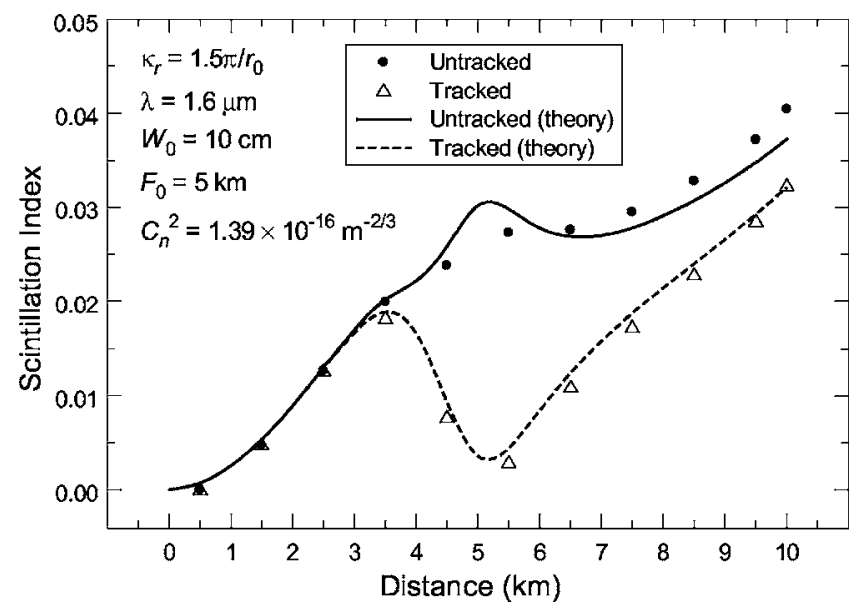

Fig. 14 On-axis scintillation index of a $10-\mathrm{cm}$ beam $F_{2}$ in both the tracked and untracked cases. 


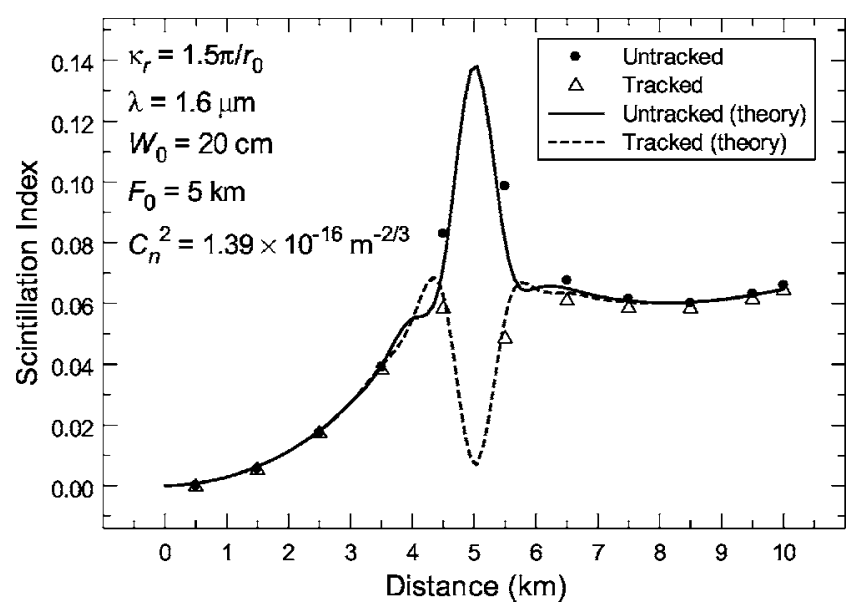

Fig. 15 Same as Fig. 14 for a 20-cm beam.

that focusing the beam at the plane of the receiver may not be the best option if the beam is not properly tracked.

\section{Discussion}

We have defined the beam wander variance $\left\langle r_{c}^{2}\right\rangle$ as that part of the squared long-term spot-size radius $W_{\mathrm{LT}}^{2}$ caused only by turbulent scale sizes on the order of the beam and larger. Thus, it is not quite the same as the variance of the centroid displacement, as our analysis here has shown, but is more general in that it also includes movement of the hot spot within the beam. Nonetheless, the variance $\left\langle r_{c}^{2}\right\rangle$ is a simple multiple of the centroid variance $\left\langle\beta_{c}^{2}\right\rangle$.

Failure of the Rytov approximation in the $D_{1}$ region was originally pointed out by Charnotskii, ${ }^{6}$ but has been discussed more recently by Baker ${ }^{5}$ in terms of two Fresnel parameters that can be used to define the boundary of this region. In the current paper, we have noted two other (equivalent) criteria that can also be used to estimate the boundary of $D_{1}$, namely, the free-space longitudinal phase shift and the excess of kurtosis, the latter of which identifies the non-Gaussian behavior of the mean irradiance profile within this region. Deviation of the mean irradiance profile from a Gaussian shape can also be observed by simply comparing the theoretical expressions (23) and (24). That is, the Gaussian function (23) is an excellent approximation to the mean irradiance result of the Rytov theory (24) except within the $D_{1}$ region, as illustrated in Figs. 8 and 9. Although we have not discussed such cases in this paper, this last criterion also applies in the case of an uplink collimated beam to space. In particular, the on-axis scintillation index of an uplink beam to space begins to increase over that predicted by Rytov theory when the mean irradiance deduced from Rytov theory can no longer be approximated by a Gaussian profile (23).

Gaussian beams propagating through the atmosphere are usually characterized by the first two moments of the irradiance in simulation studies. The first moment is related to the beam center of gravity, whereas the second is related to the beam $1 / e^{2}$ irradiance radius. Nevertheless, this last assertion holds true only for ranges outside the $D_{1}$ region. That is, because the theory for beam spot size has been developed for the $1 / e^{2}$ radius of a purely Gaussian beam, the values obtained for the short-term and long-term beam radii by the method of drawing a circle that contain $86.47 \%$ of the beam energy match better with the theoretical values within the $D_{1}$ region than do values obtained by the method of second moment (recall Figs. 7 and 8). This can be attributed to the fact that the method of circles defines the radius of an equivalent Gaussian beam that carries the same amount of energy within the circular area of that radius, whereas the circle area defined by the $2 \sigma$ radius no longer contains $86.47 \%$ of the total beam energy when the profile is non-Gaussian. Consequently, we believe that the $86.47 \%$ circle method is more suitable for comparison with theory.

Also, the deviation from Gaussian shape makes necessary the use of higher-order moments to properly characterize the properties of the beam. For example, we show in a companion paper $^{8}$ that the coefficient of skewness defined by the third central moment is related to movement of the hot spot away from the beam centroid, reaching its maximum value near the geometric focus of the beam. In the present paper, we have shown that the coefficient of kurtosis excess, defined by the fourth central moment, is an additional indicator of the non-Gaussian nature associated with both the long-term and short-term mean irradiance profiles of focused beams. In the $D_{1}$ region close to the geometric focus, the kurtosis excess may reach large values, which we believe explains the strong differences observed between the values of the scintillation index of an untracked and a tracked beam.

Generally speaking, as the kurtosis excess grows, there will be larger differences in the scintillation index between the untracked and tracked beams; i.e., as a consequence of the deviation of the mean profile from the Gaussian shape, the effect of beam wander in the scintillation index of the untracked beam increases. Conversely, as the kurtosis excess begins to vanish at distances beyond the geometric focus, there is little difference in the scintillation index between the untracked and tracked beams (e.g., see Fig. 15). As a consequence, kurtosis becomes a fundamental parameter in focused beams, since it can be considered as an indicator of the need to track the beam to reduce its scintillation index. Last, the values obtained in the scintillation index for untracked beams suggest that focusing the beam at the plane of the receiver may not be the best option if the beam is not properly tracked.

\section{References}

1. J. R. Kerr and J. R. Dunphy, "Experimental effects of finite transmitter apertures on scintillations," J. Opt. Soc. Am. 63, 1-8 (1973).

2. R. Esposito, "Power scintillations due to the wandering of the laser beam," Proc. IEEE 55, 1533-1534 (1967).

3. D. L. Fried, "Statistics of laser beam fade induced by pointing jitter," Appl. Opt. 12, 422-423 (1973).

4. P. J. Titterton, "Power reduction and fluctuations caused by narrow laser beam motion in the far field," Appl. Opt. 12, 423-425 (1973).

5. G. J. Baker, "Gaussian beam weak scintillation: low-order turbulence effects and applicability of the Rytov method," J. Opt. Soc. Am. A 23, 395-417 (2006).

6. M. I. Charnotskii, "Asymptotic analysis of finite beam scintillations in a turbulent medium," Waves Random Media 4, 243-273 (1994)

7. L. C. Andrews and R. L. Phillips, Laser Beam Propagation through Random Media, 2nd ed., SPIE Optical Engineering Press, Bellingham, WA (2005)

8. J. Recolons, L. C. Andrews, and R. L. Phillips, "Analysis of beam wander effects for a horizontal-path propagating Gaussian-beam wave: collimated beam case" (unpublished). 
9. R. G. Lane, A. Glindemann, and J. C. Dainty, "Simulation of a Kolmogorov phase screen," Waves Random Media 2, 209-224 (1992).

10. R. Frehlich, "Simulation of laser propagation in a turbulent atmosphere," Appl. Opt. 39, 393-397 (2000)

11. J. Recolons and F. Dios, "Accurate calculation of phase screens for the modeling of laser beam propagation through atmospheric turbulence," Proc. SPIE 5891, 51-62 (2005).

12. L. C. Andrews, R. L. Phillips, R. J. Sasiela, and R. R. Parenti, "Strehl ratio and scintillation theory for uplink Gaussian-beam waves: beam wander effects," Opt. Eng. 45, 076001 (2006).

13. J. H. Churnside and R. J. Lataitis, "Wander of an optical beam in the turbulent atmosphere," Appl. Opt. 29, 926-930 (1990).

14. R. L. Fante, "Electromagnetic beam propagation in turbulent media," Proc. IEEE 63, 1669-1692 (1975).

15. F. Dios, J. A. Rubio, A. Rodriguez, and A. Camerón, "Scintillation and beam wander analysis in an optical ground station-satellite uplink," Appl. Opt. 43, 3866-3873 (2004).

16. G. J. Baker and R. S. Benson, "Gaussian beam scintillation on ground to space paths: the importance of beam wander," Proc. SPIE 5550, 225-235 (2004).

17. G. J. Baker and R. S. Benson, "Gaussian-beam weak scintillation on ground-to-space paths: compact descriptions and Rytov-method applicability," Opt. Eng. 44, 106002 (2005).

18. R. J. Sasiela, Electromagnetic Wave Propagation in Turbulence, Springer, New York (1994).

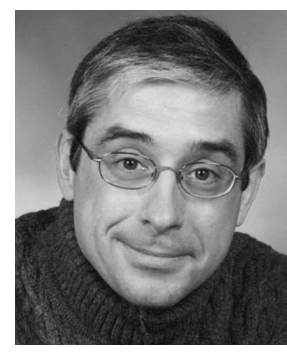

Jaume Recolons received his BS and MS degrees in physics from the University of Barcelona, Spain, in 1988 and his PhD degree in applied science from the Polytechnic University of Catalonia, Spain, in 1991. He is currently an associate professor of electromagnetic fields at the Polytechnic University of Catalonia. His current research interests include laser propagation in the atmosphere and atmospheric turbulence.

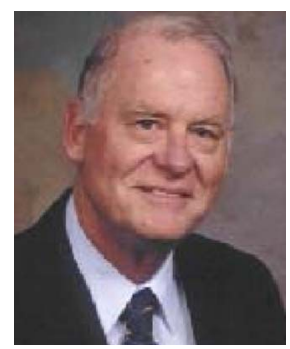

Larry C. Andrews is a professor of mathematics at the University of Central Florida and an associate member of the College of Optics/CREOL. He is also an associate member of the Florida Space Institute (FSI). Previously, he held a faculty position at TriState University and was a staff mathematician with the Magnavox Company, antisubmarine warfare (ASW) operation. $\mathrm{He}$ received a doctoral degree in theoretical mechanics in 1970 from Michigan State University. He has been an active researcher in optical wave propagation through random media for more than 25 years and is the author or coauthor of ten textbooks on topics of differential equations, boundary value problems, special functions, integral transforms, wave propagation through random media, and mathematical techniques for engineers. Along with wave propagation through random media, his research interests include special functions, random variables, atmospheric turbulence, and signal processing.

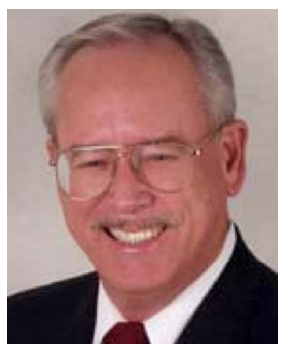

Ronald L. Phillips is director of the Florida Space Institute (FSI) and a professor in the Department of Electrical and Computer Engineering at the University of Central Florida. He is also a member of the Department of Mathematics and an associate member of the College of Optics/CREOL. $\mathrm{He}$ has held positions on the faculties at Arizona State University and the University of California, San Diego. He received a doctoral degree in electrical engineering in 1970 from Arizona State University. He has been an active researcher in wave propagation through random media for more than 28 years. He was awarded a Senior NATO Postdoctoral Fellow in 1977 and the American Society for Engineering Education 1983 Medal for outstanding contributions in research. He is coauthor of two textbooks on wave propagation through random media and mathematical techniques for engineers. In addition to optical wave propagation, his research interests include optical communications and imaging through atmospheric turbulence. 